\title{
Graphene characterization based nano dipole antennas design for nano network wireless communication applications
}

\author{
Arti Shrivastava*, S.K. Jain ${ }^{\#}$, Shobha Jain ${ }^{\circledR}$ \\ ${ }^{* \#}$ Department of Electronics and Telecommunication Engineering, Shri G.S. Institute of Technology \& Science, \\ Indore (M.P.),India \\ ${ }^{\circledR}$ Department of Mathematics, S.V.V.V, Indore, (M.P.), India
}

(Received 12 April 2021; Accepted 27 June 2021)

DOI: https://doi.org/10.36224/ijes.140202

\begin{abstract}
Graphene-enabled nano antennas constitute a novel paradigm which is a current trend of research among designers. Graphene-based nano-antennas with nanometer in size have shown to radiate electromagnetic waves at the terahertz (THz) frequency band. Proposed research activity is an attempt of Terahertz Nano dipole antenna design through the Finite Difference Time Domain (FDTD) based numerical simulation software, i.e. CST. Graphene material as adipole antenna has been explored to get a radiation performance. Before hand absorption, reflection and transmission characteristics of the grapheme layer has been explored. Designed antenna is analyzed in terms of the reflection coefficient, and radiation pattern etc. An attempt has been done for resonance study of graphene based nano dipole antennas, in that patch antenna length parameteris changed and performance is observed. The impact of most crucial parameter, i.e., chemical potential on the grapheme radiation performance has been analyzed in terms of reflection coefficient. Proposed antenna may be suitable for wireless nano communication applications.
\end{abstract}

Keywords: Terahertz antennas, computer simulation technology, microstrip dipole antenna, reflection coefficient, radiation pattern

\section{Introduction}

As per the current trend, researchers have been attempting hard to design and develop the antennas in $\mathrm{RF}$ and microwave frequency band for various applications. But looking in to the need of future applications having required enormous bandwidth and data speed, they are shifting focus towards untouched frequency band i.e. terahertz $(\mathrm{THz})$. Terahertz $(\mathrm{THz})$ band covering frequency range of the order of $0.1 \mathrm{THz}$ to $10 \mathrm{THz}$ is being utilized in many field such as spectroscopy, high-resolution radar, imaging and sensing and detection etc.[1] Recently the RF researchers and designers have shifted their focus to explore the $\mathrm{THz}$ band for the possibility of wireless nano communications applications. However yet challenging given the large propagation losses, lack of mature devices and circuits for terahertz operation as well as dirth of advanced electronic and photonic materials. Therefore there is need of a new material and devices at terahertz frequency. In search of efficient solutions to these problems, graphene appears as an excellent candidate for the implementation of new devices like antennas etc. Due to its unique thermal, mechanical and electrical properties at terahertz $(\mathrm{THz})$ frequencies [2], graphene has attracted huge interests among researchers and designers. Because of ability to support transverse magnetic surface plasmonic polariton (SPP) modes as well as gapless energy spectrum, researchers are putting attempt to design, and develop the grapheme based nano antennas [3-4]. Since antenna is a critical component for wireless communication application, therefore

\footnotetext{
${ }^{*}$ Corresponding author

Email address: arti17shrivastava@gmail.com(Arti Shrivastava)

ISSN 0976 - 6693. C2021 SCMR All rights reserved.
} 
effective design and development of nano antennas is a core issue among the researchers in the terahertz frequency band applications.

\section{Literature review}

Very few researchers have attempted antenna designs using grapheme material. Graphene may replacement of copper in antenna design due to low loss, light weight, low corrosive behavior, cheaper, less energy consuming, higher efficiency and reduced $\mathrm{CO} 2$ emission through it. In this connection, graphene-based Yagi-Uda antenna with reconfigurable radiation patterns and circularly polarized graphene based transmit array in terahertz frequency band has been already suggested in [2-3]. Fabrication and characterization of near-field communication (NFC) devices based on highly flexible, carbon-based antennas composed of stacked graphene multilayers has been presented by authors in [4]. The simulation results of [5] demonstrated that the designed structure can provide excellent properties in terms of dual wide-band performance, frequency-reconfiguration by applying different voltages on the graphene. The effect of the temperature and relaxation time on the antenna performance, and use of graphene for reconfigurable, miniaturized, and efficient terahertz (THz) antennas and associated feeding networks development have been investigated in [6]. Also an attempt has been done to identify current research trends and mid- and long-term challenges and prospects. Authors presented graphene-based CNT nano-antennas, ordinary dipole, fractalized dipole, spiral, bow-tie and log-periodic configurations, miniaturized and tunable antennas, plasmonic graphene antennas in the terahertz band for electromagnetic nano communications in [7-10]. The graphene based antennas showed significant improvements for most parameters of antenna than that of the conventional antenna. In [11], the performance of a graphene-based nano-patch antenna in transmission and reception has been analyzed numerically. The resonance frequency of the nano-antenna is calculated as a function of its length and width, both analytically and by simulation. Work done by authors in [12-13], provides a brief overview of terahertz antenna issues from large scale reflectors to multipixel imaging systems, as well as special type of tunable feeding point slot antenna with butterfly type slot. A novel and well-performed design of terahertz horn antenna with microstrip feed and several configurations of antennas based on graphene is presented by the authors [14-15]. In [16], a method has been proposed to design microstrip antennas at terahertz $(\mathrm{THz})$ frequencies which improved the antenna gain, bandwidth and fabrication tolerance. The measured and the simulation results were in good agreement. Two configurations of smart graphene antennas has been reported through the work [17], in which either the radiation pattern of the antenna or the backscattering of the periodic metallic arrays is controlled by DC biases. The combination of metallic structures and graphene, which has gate-voltage dependent optical properties, is emerging as a possible platform for electrically controlled plasmonic devices. In [18],control of antennas using graphene as an electrically tunable load in the nanoscale antenna gap is demonstrated. Through the experiments, electrical tuning of graphene-loaded antennas over a broad wavelength range of $650 \mathrm{~nm}$ in the mid-infrared (MIR) region was demonstrated. An equivalent circuit model was proposed to analyze the tuning behavior of graphene-loaded antenna pairs and an analytical expression was derived for the tuning range of resonant wavelength.

From the above brief review report, it is very clear that grapheme based terahertz antenna design is in infant stage among researchers and very little work has been reported sofar.

\section{Problem formulation}

In usual practice of rectangular patch antenna design, RF researchers have been using numerous substrate with dielectric constant ranging from $2.2<\varepsilon_{\mathrm{r}}>12$ to achieve good performance of antenna. 
Copper patch with some prespecified shape was being etched on the dielectric substrate having specific height. The lower side of the dielectric sheet is having the polish of copper layer and acting as a ground plane, which serves to get the broadside radiation pattern through antenna. In order to excite the antenna (copper microstrip patch), suitable feeding arrangement can be applied like coaxial feed, inset feed or aperture coupled feed. The typical structure of the microstrip patch antenna can be understood with following Figure 1. In the proposed nano dipole antenna, we have employed graphene material layer in place of copper and designed and performance of the antenna was tested using numerical simulator. The graphene based terahertz nano dipole antennas are shown in the Figurel (b). Before hand, we have attempted to characterize the graphene for its conductivity, reflection, transmission as well as absorption behavior with respect to terahertz frequency range.

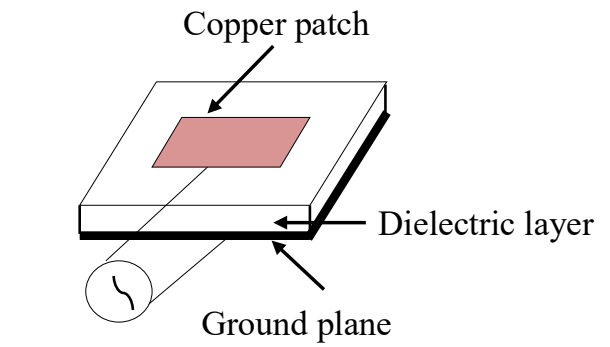

RF source

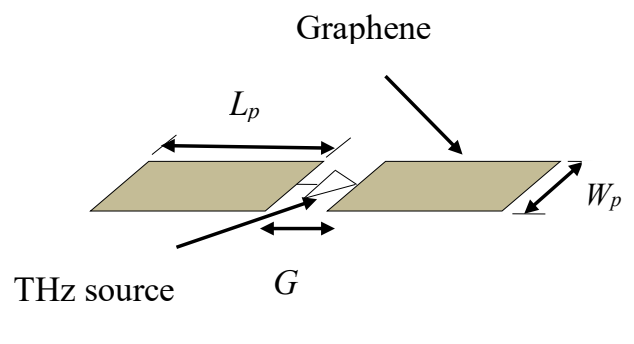

(b)

Figure 1: (a) Typical RF microstrip antenna (b) Proposed terahertz dipole antenna

\subsection{Graphene modeling}

The graphene monolayer is a two-dimensional material composed of carbon atoms bonded in hexagonal structures. It can be represented by an infinite sheet with surface conductivity, which can be modeled via Kubo formula [11]. It consists of an intraband contribution and an interband contribution as shown in Equation (1) and Equation (2)

$$
\begin{gathered}
\sigma\left(\omega, \mu_{c}, \Gamma, \mathrm{T}\right)=\sigma_{\text {intra }}(\omega)+\sigma_{\text {inter }}(\omega)(1) \\
\sigma\left(\omega, \mu_{c}, \Gamma, T\right)=-j \frac{e^{2} K_{B} T}{\pi h^{2}\left(\omega-j \tau^{-1}\right)}\left(\mu_{c} / K_{B} T+2 \ln \left(\exp \left(-\mu_{c} / K_{B} T\right)+1\right)\right) \\
-\frac{j e^{2}}{4 \pi h} \ln \left[\frac{2\left|\mu_{c}\right|-\left(\omega-j \tau^{-1}\right) \bar{h}}{2\left|\mu_{c}\right|+\left(\omega-j \tau^{-1}\right) \bar{h}}\right]
\end{gathered}
$$

Where $j$ is the imaginary unit. The electron charge, $e=1.60217657 \times 10^{-19} \mathrm{C}$; The reduced Plank constant, $h=6.62606957 \times 10^{-34} / \Omega \mathrm{eV} / \mathrm{s}$; Permittivity, $\varepsilon_{0}=8.85418781762 \times 10^{-12} \mathrm{~F} / \mathrm{m}$; The Boltzmann's constant, $K_{B}=1.3806488 \times 10^{-23} \mathrm{~m}^{2} \mathrm{~kg} \mathrm{~s}^{-1} \mathrm{~K}^{-1}$; The temperature, $T=300 \mathrm{~K}$; The chemical potential, $\mu_{c}=$ $0 \mathrm{eV}$ to $1 \mathrm{eV}$; The operating angular frequency, $\omega(=2 \pi \times f) \mathrm{Hz}$; Scattering rate, $\Gamma,=1 / 2 \tau$ (represents its loss mechanism) and, The relaxation time, $\tau=0.1$ ps. The value of $\tau$ in previous literature ranges between $10^{-11}$ and $10^{-14}$. In our work, we have utilized $\tau=1 \times 10^{-12} \mathrm{sec}$. The two terms of the surface conductivity are calculated using Equation (1) and Equation (2). The first term (intraband term) dominates the value of total conductivity in the range of frequency below $5 \mathrm{THz}$, whereas the second term (interband term) has no significant effect on the total surface conductivity within this band. Impact of changing graphene chemical potential $\mu_{c}$ on the surface conductivity is big prospectus for terahertz antenna design (Figure 1 (b)). It depends on the carrier density, which can be controlled by gate voltage, 
electric bias field, or chemical doping. Increasing $\mu_{c}$ leads to the increase in graphene surface conductivity, which shifts antenna resonances to higher frequencies. The shifting of antenna resonance, due to changing $\mu_{c}$ enhances flexibility for the design of tuneable antennas, especially within the $\mathrm{THz}$ band. The variation of $\mu_{c}$ enables the resonant frequency tunability feature. The value of the chemical potential can be electrically controlled by varying the bias voltage (gate voltage, $V_{g}$ ) on the graphene layer. Graphene presents excellent conditions for the propagation of Surface Plasmon Polaritons (SPP), waves guided along a metal-dielectric interface which are generated by an incident high-frequency radiation.

\subsection{Graphene characterization}

Graphene is a one of the most important elements being explored for engineering applications by the researchers since it is discovered in 2004. It is one atom 0.3 nanometer thick layer of carbon atoms having covalent bond with 3 other carbon atoms, and is arranged in a honeycomb (hexagonal) lattice 2 D structure. It is having some remarkable thermal, electrical, chemical and mechanical properties, because of that it has got place in various engineering applications. At room temperature, graphene has a thermal conductivity much higher than other carbon allotrope's; it is one of the most important properties for graphene based electronics. Mechanically graphene is a very light, strong and flexible material, which is stronger than a diamond and steel. As per electronic properties, graphene deposited silicon wafer is able to absorb more than $2.5 \%$ of the light. Chemical properties for graphene are also very unique. Chemically graphene has weekly attached atoms, which can act as acceptors for other atoms maintaining a high conductivity, which leads to its applications in few electronics devices [19].

While from the perspective of frequency, the contrasting of the conductivities of different chemical potential $\left(\mu_{c}\right)$ become smaller with frequency increasing. The 1-4 THz band shows larger conductivity distinction than the higher frequencies and it might be good choice for some designing in this frequency range. The conductivities of the graphene are owing to the carrier density, which is controlled by the $\mu_{c}$. The conductivity for $\mu_{c}=0.01 \mathrm{eV}$ is very small and close to zero due to low carrier density. In this case, the graphene acts as a thin dielectric media, which can be treated as transparency for EM wave. The conductivity of the graphene is large for large $\mu_{c}$ owing to larger carrier density, then, the graphene supports surface plasmon-polaritons (SPPs), which would be used to confine the incident EM wave absorption and lead to absorption.

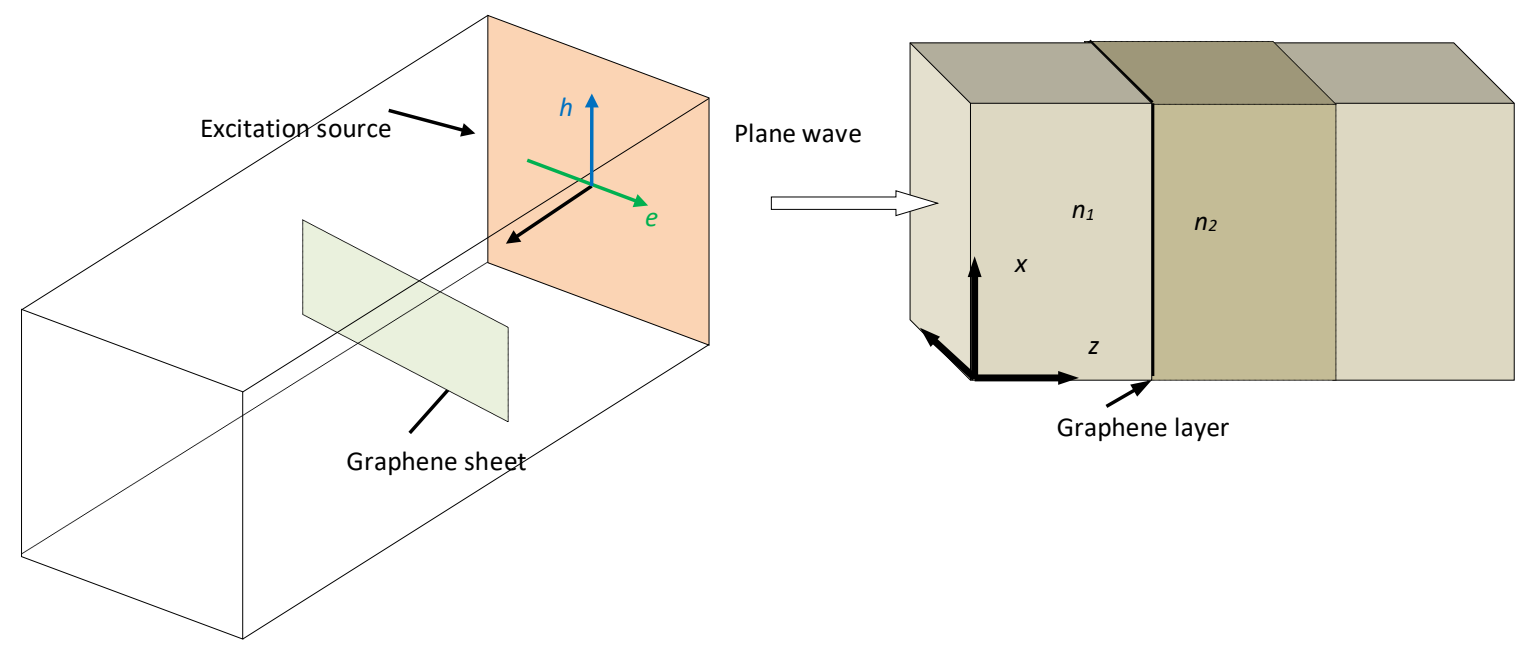

Fig. 2. A graphene sheet normally illuminated by a plane wave. 
Here, we consider a graphene sheet normally illuminated by a plane wave as shown in Fig. 2. The graphene sheet is laid in the xoy-plane, while the $z>0$ space is filled with medium 1 characterized by $\varepsilon_{1}$ and $\mu_{1}$, and the medium 2 in $\mathrm{z}<0$ space is $\varepsilon_{2}$ and $\mu_{2}$. Then, the reflection coefficient, the transmission coefficient and the absorption are [20] as follow.

$$
\begin{aligned}
& r=\frac{\eta_{1}-\eta_{2}-\sigma_{s} Z_{0}}{\eta_{1}+\eta_{2}+\sigma_{s} Z_{0}} \\
& t=\frac{2 \eta_{1}}{\eta_{1}+\eta_{2}+\sigma_{s} Z_{0}} \\
& A=\frac{4 \xi}{(1+\gamma+\xi)^{2}+\xi^{2}}
\end{aligned}
$$

Where $\eta_{1}=\sqrt{\mu_{1 r} \varepsilon_{1 r}}$ and $\eta_{2}=\sqrt{\mu_{2 r} \varepsilon_{2 r}}$ are the refraction coefficients of the medium 1 and medium 2, respectively. The $\mathrm{Z}_{0}=120 \pi \mathrm{Ohm}$ is the free-space impedance. Also

$$
\begin{aligned}
& \gamma=\frac{\eta_{2}}{\eta_{1}} \\
& \xi^{\prime}+i \xi^{\prime \prime}=\left(\sigma_{s}^{\prime}+i \sigma^{\prime \prime}{ }_{s} Z_{0} / \eta_{1}\right.
\end{aligned}
$$

For the special case $\eta_{1}=\eta_{2}=\eta_{0}=1$, where $\eta_{0}=\sqrt{\mu_{0 r} \varepsilon_{0 r}}$ is the refraction coefficient of the air.In that case, the reflection coefficient, the transmission coefficient and the absorption coefficient; $r, t$ and $A$ of Equations 3, 4 and 5 respectively can be written as

$$
\begin{aligned}
& r=\frac{-\sigma_{s} Z_{0}}{2+\sigma_{s} Z_{0}} \\
& t=\frac{2}{2+\sigma_{s} Z_{0}} \\
& A=\frac{4 \xi}{(2+\xi)^{2}+\xi^{2}} \\
& \text { Or } A=\frac{4 \sigma_{s}^{\prime} Z_{0}}{\left(2+\sigma_{s}^{\prime} Z_{0}\right)^{2}+\left(\sigma^{\prime \prime}{ }_{s} Z_{0}\right)^{2}}
\end{aligned}
$$

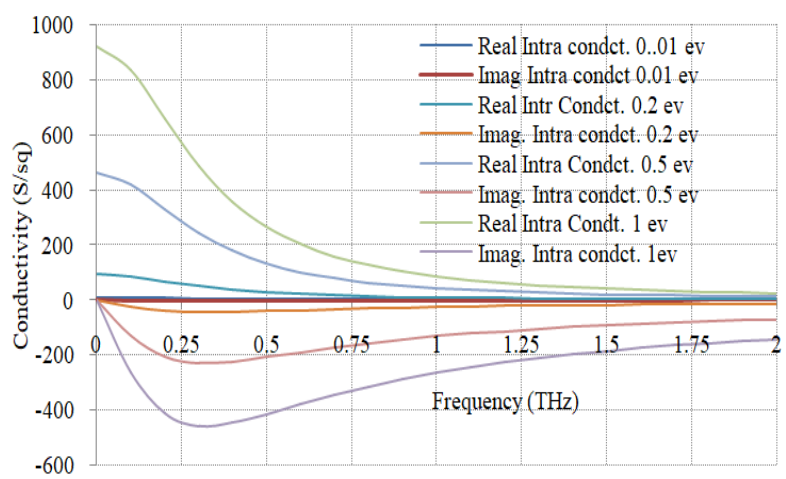

(a)

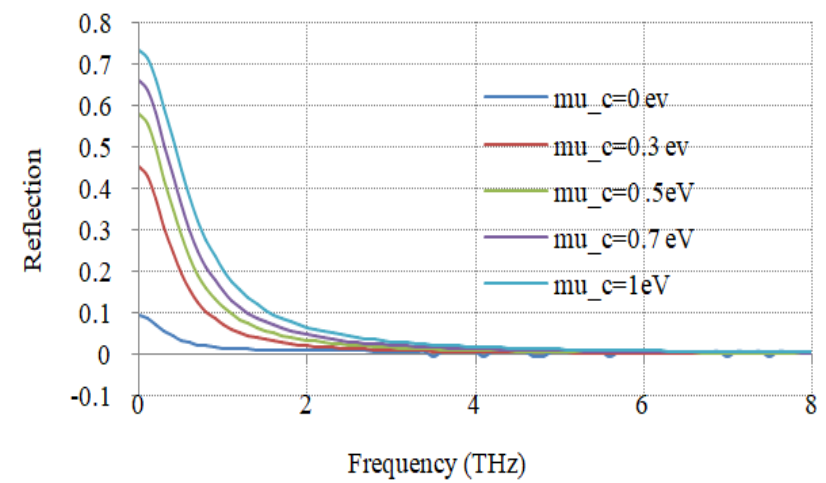

(b) 


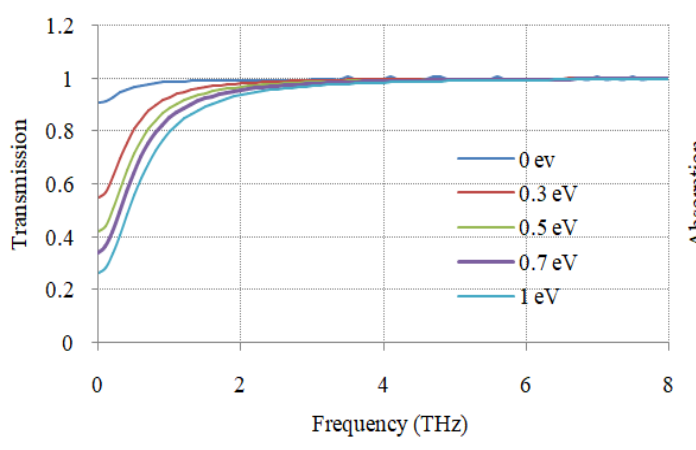

(c)

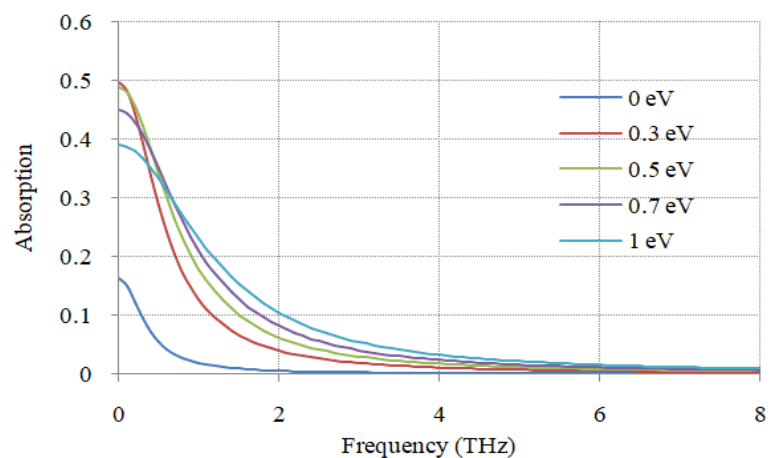

(d)

Fig.3. Grapheme (a) intra band conductivity (real and imaginary part w. r. $\mathrm{t} \mu_{c}$ ) (b) reflection(c) transmission (d) absorption coefficient plot.

From Fig. 3 (a), the conductivity $\sigma_{s}$ of the graphene is zero for $\mu_{c}=0.01 \mathrm{eV}$, then transmission coefficient $t=1$, the reflection coefficient $r=0$, and the absorption $A=0$ (Eqs. (8) - (9)). In this case, the graphene sheet is transparent to the electromagnetic wave. Also from same Equation, (Eqs. (8) - (9)), a large conductivity $\sigma_{s}$ (due to large $\mu_{c}$ ) results in large reflection coefficient, $r$ and small transmission coefficient, $t$. From Eq. (10), the absorbance would be very small as the imaginary part $\sigma_{\text {real }}$ is larger than the real part $\sigma_{\text {imag, }}$ as also indicated in Fig. 3. Therefore, the graphene operates as semi-conductor with large $\mu_{c}$. To verify the above discussion, the reflectance, transmission and absorption of the graphene sheet in terms of the chemical potential $\left(\mu_{c}\right)$ are calculated and plotted in Fig. 3 (b), 3 (c) and 3 (d), respectively. As the conductivity of the graphene increased with $\mu_{c}$ increasing (Fig. 2 (a)), the graphene acts more like a metal, so that a larger reflectance is obtained with higher $\mu_{c}$ as demonstrated in Fig. 2(b). Correspondingly, the transmission decreased with $\mu_{c}$ increasing as shown in Fig. 2(c). It is also found that, the $\mu_{c}=0 \mathrm{eV}$ of the graphene sheet leads to a reflectance almost equals 0 and a transmission almost equals 1 in the 1-2 THz band, which verify the aforementioned conjecture that the graphene with $\mu_{c}=0 \mathrm{eV}$ acts as a very thin dielectric media. However, for the non-zero $\mu_{c}$, the reflectance is increased while the transmission is decreased with $\mu_{c}$ increasing. The absorptions presented in Fig. 2 (d) are also sufficient very small for all the $\mu_{c}$ values to enhance plasmonic resonances.

\section{Results and discussion}

The proposed terahertz nano dipole antenna has been designed and simulated using CST Microwave Studio 2017. Before hand the intraband and interband conductivity has been plotted for terahertz frequency range with different chemical potential as shown in Figure 3 (a). From the Figure 3(a), it can be easily observed that intraband conductivity (I term) dominates the value of total conductivity in the range of frequency below $5 \mathrm{THz}$, whereas the interband conductivity (II term) has no significant effect on the total surface conductivity within this band. Nano dipole has been excited using discrete port having $1300 \mathrm{ohm}$ input impedance values. The reflection coefficient performance plot for chemical potential $0.12 \mathrm{eV}$ is shown in the Figure 4, where resonance condition is achieved for $1.08 \mathrm{THz}$ frequency. Directivity of the proposed nano dipole is nearly $2 \mathrm{dBi}$ as shown in Figure 5 (from 2-d and 3$\mathrm{d}$ far field plots).From the $\mathrm{E}$ and $\mathrm{H}$-field (2-d) polar plot, same value of directivity can be observed. The numerical values of the grapheme and nano dipole antenna parameters are listed in Table 1. 
Table 1: Parameters of grapheme nano dipole antenna

\begin{tabular}{|l|l|l|}
\hline S.N. & Parameters & Values \\
\hline 1 & Graphene thickness & $0.35 \mathrm{~nm}$ \\
\hline 2 & Graphene chemical potential, $\mu_{c}$ & $0.12 \mathrm{eV}$ \\
\hline 3 & Temperature, $T$ & $300 \mathrm{~K}$ \\
\hline 4 & Relaxation time, $\tau$ & $1 \mathrm{ps}$ \\
\hline 5 & Feed gap, $G$ & $2000 \mathrm{~nm}$ \\
\hline 6 & Feed impedance & $2000 \Omega$ \\
\hline 7 & Dipole length, $L_{p}$ & $25000 \mathrm{~nm}$ \\
\hline 8 & Dipole width, $W_{p}$ & $10000 \mathrm{~nm}$ \\
\hline
\end{tabular}

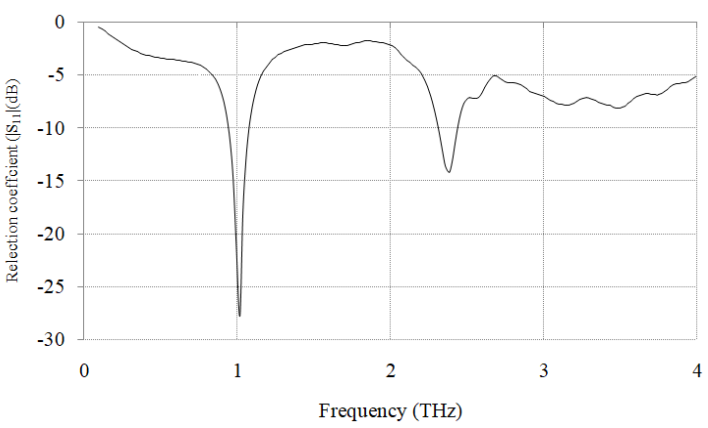

Figure 4: Reflection coefficient plot of nanodipole antenna

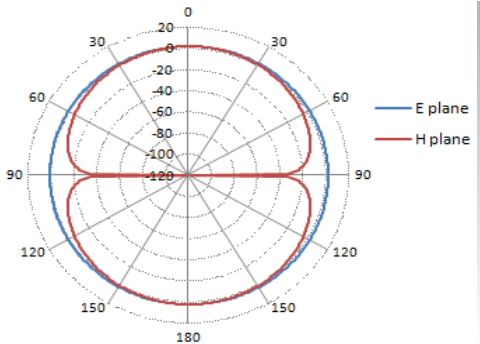

(a)

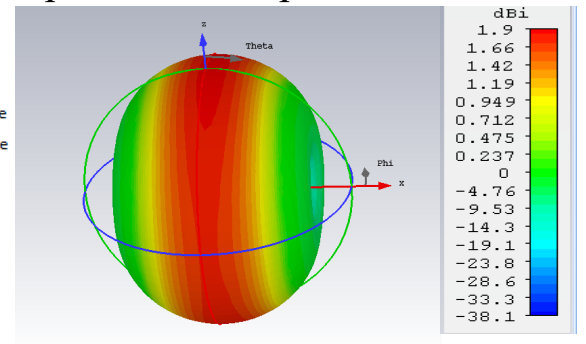

(b)

Figure 5: (a) 2-d, $E$ and $H$ plane pattern (b) 3-d, far field radiation pattern of nano dipole

\section{Conclusion}

Graphene-based nano-antenna has been explored which can work for terahertz frequency range and may be useful for nanosystems to transmit and receive information. In this work, we proposed a low profile model of a graphene-based nano-dipole antenna, and performance is estimated by means of simulation. Modeling starts with analyzing the conductivity of graphene numerically. Also graphene characterization has been done through its transmission, reflection and absorption coeffcient. Electromagnetic CST Microwave Studio simulator 2017 has been used for this purpose. The design uses a discrete feed source in order to obtain proper impedance matching between the nanodipole and the feed. The proposed antenna is resonant at a frequency of $1.05 \mathrm{THz}$ with corresponding return reflection coefficient of the order of $-28 \mathrm{~dB}$. The designed antenna has the satisfactory level of directivity of the order of $2 \mathrm{dBi}$ at the resonant frequency. 


\section{Acknowledgment}

Authors are thankful to the AICTE New Delhi (India) funded advanced RF and Microwave MODROB laboratory of SGSITS Indore (MP) India for supporting this research activity.

\section{References}

[1] Jha K.R., Singh G., Terahertz planar antennas for next generation communication, Springer International Publishing Switzerland 2014.

[2] Wu Y., Qu M., Jiao L., Liu Y., Ghassemlooy Z., Graphene-based yagi-uda antenna withreconfigurable radiation patterns, AIP Advances, June 2016. Pp. 134-139 doi.org/10.1063/1.4953916.

[3] Malhat H.A., Zainud Deen S.H., and Gaber S.M., Graphene based transmit array for terahertz applications, Progress in Electromagnetics Research M, vol. 36, 2014, pp. 185-191.

[4] Scidà A., Haque S., Treossi E., Robinson A., Smerzi S., Ravesi S., Borini S., Palermo V., Application of grapheme based flexible antenna in consumer electronic devices", Research Materials Today Elsevier, vol.21, no.3, 2018.

[5] H lali A., Houaneb Z., and Zairi H., Dual-band reconfigurable graphene-based patch antenna in terahertz band: design, Progress In Electromagnetic Research c, vol. 87, 2018, pp.213-226.

[6] Serrano D.C., Diaz J. S. G., Graphene-based antennas for terahertz systems: a review, Forum for Electromagnetic Research Methods and Application Technologies (FERMAT), vol. 8, no.7 pp. 1-25.

[7] Jornet J. M., Akyildiz I. F., Graphene-based nano-antennas for electromagnetic nano communications in theterahertz band, IEEE Proceedings of the Fourth European Conference on Antennas and Propagation, 2010, pp.12-16.

[8] Biabanifard M., Biabanifard S., Hosseini S. J. and Jahanshiri A.,Design and comparison of terahertz graphene antenna: ordinary dipole, fractal dipole, spiral, bow-tie and log-periodic, Eng Techno Open Acc. Journal, 2018; vol. 2, issue 2. doi: 10.19080/ETOAJ.2018.02.555584

[9] Abadal S., Hosseininejad S. E., Aparicio A. C., and Alarcón E., Graphene-based terahertz antennas for areaconstrained applications", 40th International Conference on Telecommunications and Signal Processing (TSP), 2017.

[10] Gatte M.T., Soh P.J., Rahim H.A., Ahmad R. B., and Malek M.F. A., The performance improvement of THz antenna via modeling and characterization of doped graphene, Progress In Electromagnetics Research M, vol. 49, 2016, pp. 21-31.

[11] Llatser I., Kremers C., Chigrin D.N., Jornet J.M., Lemme M. C., Aparicio A. C., and Alarcon E., Characterization of graphene-based nano-antennas in the terahertz band. 6th European Conference on Antennas and Propagation (EUCAP), 2012.

[12] Siegel P. H., Maagt P. and Zaghloul A. I., Antennas for terahertz applications, IEEE Antennas and Propagation Society International Symposium, 2006. doi: 10.1109/APS.2006.1711074.

[13] Zhang J., Gan X., Yang B., Wang S., Gao K., and Zheng Z., Tunable feeding point THz antenna with butterfly type slot based on ceramic material substrate, 40th International Conference on Infrared, Millimeter, and Terahertz waves (IRMMW-THz), 2015.

[14] Li Z., Fan Y., Wang X., and Tang X., A design of on-chip terahertz horn antenna with microstrip feed, IEEE International Conference on Integrated Circuits and Microsystems (ICICM), 2016.

[15] Dragoman M., Muller A. A., Dragoman D., Coccetti F., and Plana R., Terahertz antenna based on graphene, Journal of Applied Physics, 210. doi: 10.1063/1.3427536.

[16] Rabbani M. S., Ghafouri-Shiraz H., Improvement of microstrip antenna's gain, bandwidth and fabrication tolerance at terahertz frequency bands, IET, Wideband and Multi-Band Antennas and Arrays for Civil, Security \& Military Applications, 2015. doi: 10.1049/ic.2015.0146.

[17] Aldrigo M., Dragoman M., and Dragoman D., Smart antennas based on grapheme" Journal of Applied Physics 116, 2014. doi: 10.1063/1.4895739.

[18] Yao Y., Kats M.A., Genevet P., Yu N., Song Y., Kong J., and Capasso F., Broad electrical tuning of graphene-loaded plasmonic antennas, Nano Letter, 2013, pp.1257-1264. dx.doi.org/10.1021/nl3047943. 
[19] Dash S., Patnaik A., Material selection for THz antennas, Microwave and Optical Technology Letters · 2018, pp.1183-1187.

[20] Peng L., Li X., Gao X., Jiang X., and Li S., Methodology for the design of a multi-functional device with switchable absorption and polarization conversion modes by graphene and metallic metasurfaces, Optical Materials Express, 2019, pp. 687-705. 